\title{
Experimental Study on Piezoelectric Energy Harvesting from Vortex-Induced Vibrations and Wake-Induced Vibrations
}

\author{
Min Zhang ${ }^{1,2}$ and Junlei Wang ${ }^{3,4,5}$ \\ ${ }^{1}$ School of Mechanical Engineering, Shanghai Jiao Tong University, 800 Dongchuan Road, Shanghai 200240, China \\ ${ }^{2}$ Shipping and Marine Engineering College, Chongqing Jiao Tong University, Chongqing 40074, China \\ ${ }^{3}$ School of Chemical Engineering and Energy, Zhengzhou University, Zhengzhou 450002, China \\ ${ }^{4}$ Key Laboratory of Low-Grade Energy Utilization Technologies and Systems, Chongqing University, Ministry of Education of China, \\ Chongqing 400044, China \\ ${ }^{5}$ Jiangsu Engineering Research Center on Meteorological Energy Using and Control, Nanjing University of \\ Information Science \& Technology, Nanjing 210000, China \\ Correspondence should be addressed to Junlei Wang; just4pipi@126.com
}

Received 3 March 2016; Accepted 13 April 2016

Academic Editor: Rui Tang

Copyright (C) 2016 M. Zhang and J. Wang. This is an open access article distributed under the Creative Commons Attribution License, which permits unrestricted use, distribution, and reproduction in any medium, provided the original work is properly cited.

A rigid circular cylinder with two piezoelectric beams attached on has been tested through vortex-induced vibrations (VIV) and wake-induced vibrations (WIV) by installing a big cylinder fixed upstream, in order to study the influence of the different flowinduced vibrations (FIV) types. The VIV test shows that the output voltage increases with the increases of load resistance; an optimal load resistance exists for the maximum output power. The WIV test shows that the vibration of the small cylinder is controlled by the vortex frequency of the large one. There is an optimal gap of the cylinders that can obtain the maximum output voltage and power. For a same energy harvesting device, WIV has higher power generation capacity; then the piezoelectric output characteristics can be effectively improved.

\section{Introduction}

In recent years, many researchers have started the study of various replaceable and environment-friendly energy harvesting ways due to the traditional global energy and environment-protection issues. Utilizing the energy harvested from atmosphere or aeroelastic vibration to supply power for electronic devices becomes a hot-topic. Most researches have given attention to the power-supply of the lower power-consumption devices including MEMS, actuator [1,2], wireless sensor networks, and health monitoring device $[3,4]$, or seeking replacements for the batteries used on the devices which are expensive and hard to maintain [5]. The issue of harvesting aeroelastic energy from airfoils has been widely studied [6-12]. In addition, some other researches are focused on the energy conversion generated by the vortex-induced vibrations of flags or microbimorph structures [13-15].
Vortex-induced vibrations (VIV) and wake-induced vibrations (WIV) are two main types of flow-induced vibrations (FIV). VIV is a self-excited vibration; when the vortex shedding frequency is near to the natural frequency of the bluff body, "lock-in" or synchronization phenomena will happen [16], and the amplitude is decided by the mass-damping ratio. When an elastically mounted cylinder is immersed in the wake of another bluff body, WIV occurs, which is usually completely different from the VIV of a single cylinder in free stream. Researches on two cylinders of same diameter [17-20] show that proper spaces between cylinders can make downstream cylinders obtain higher amplitudes and larger vibration intervals. In addition, according to the researching results on the vibration of smaller cylinders in the wake of bigger cylinders [21, 22], when cylinders are arranged in sequence, the vibration frequency of the smaller downstream cylinder is dependent on the vortex shedding frequency of 


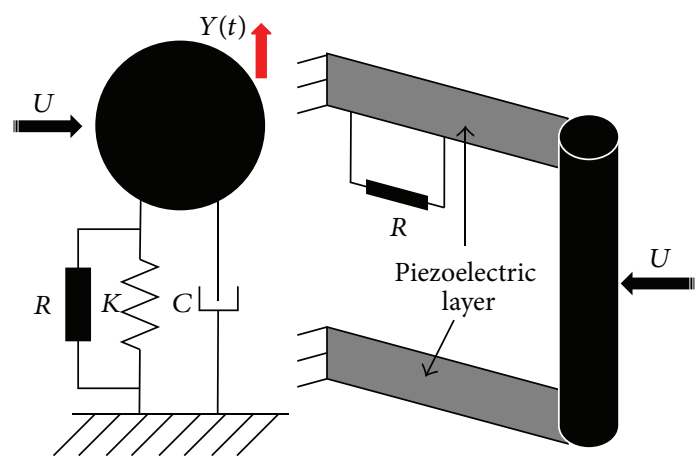

FIGURE 1: Schematic of a piezoelectric energy harvester.

the bigger upstream cylinder, and if the range of speed is bigger, then the higher amplitude can be obtained.

In order to research the harvesting of piezoelectric energy generated from VIV, Akaydin et al. [23] carried out a tunnel test on a single piezoelectric transducer equipment with the diameter of $26.7 \mathrm{~mm}$ and got the max power of $0.1 \mathrm{~mW}$. Molino-Minero-Re et al. [24] tested the VIV energy harvesting on a series of single cantilever cylindrical piezoelectric devices; these tests were made in water channel. The max power of $0.31 \mu \mathrm{W}$ was obtained at the diameter of $8 \mathrm{~mm}$. Mehmood et al. [25] calculated the energy harvested from the VIV with low Reynolds number and high mass ratio and obtained the maximum power of $10 \mu \mathrm{W}$. Zhang et al. [26] carried out a numerical study of the similar structure, which shows that the harvested level in SS and SP\&PS modes is the same with different values of load resistance.

On the basis of relevant researching results on VIV and WIV, this paper's research involved three aspects: (1) the energy harvesting of a cylinder installed with two piezoelectric cantilevers; (2) the voltage outputs of VIV under different load resistance; (3) the impacts of the space between the two circular cylinders on the voltage output under WIV of the big cylinder.

\section{The Piezoelectric Energy Harvesting Model}

As shown in Figure 1, two bimorph piezoelectric cantilevers are attached to the cylinder as elastic support; when the cylinder is suffering VIV or WIV in the transverse direction, the deformations of the cantilevers produce stress in the piezo layers. Based on the piezoelectric effect, there will be voltage produced between the piezo layers and current produced in the circuit as well. Thus the piezoelectric energy harvester can output power if there is a load in the circuit. The control equation of the system can be represented with the coupled equations of the spring supported rigid cylinder and the Gauss law $[6,9]$ :

$$
\begin{aligned}
M \ddot{Y}+2 M \xi \omega_{n} \dot{Y}+M \omega_{n}^{2} Y-\theta V & =F(t), \\
C_{e} \dot{V}+\frac{V}{R}+\theta \dot{Y} & =0,
\end{aligned}
$$

where $M$ is the mass, $\xi$ is the damping ratio, $\omega_{n}$ is the natural frequency of the structure, $F(t)$ is the lateral fluid force, $\theta$ is
TABLE 1: Parameters of the four considered configurations.

\begin{tabular}{lcc}
\hline Symbol & Parameter & Value \\
\hline$M$ & Mass $(\mathrm{g})$ & 5.8 \\
$D$ & Diameter of big cylinder $(\mathrm{mm})$ & 40 \\
$d$ & Diameter of small cylinder $(\mathrm{mm})$ & 10 \\
$L_{c}$ & Length of cylinder $(\mathrm{mm})$ & 25 \\
$L$ & Length of cantilever $(\mathrm{mm})$ & 45 \\
$b$ & Thickness of cantilever $(\mathrm{mm})$ & 15 \\
$L_{p}$ & Length of piezoelectric layer $(\mathrm{mm})$ & 30 \\
$h_{s}$ & Thickness of coppery base layer $(\mathrm{mm})$ & 0.2 \\
$h_{p}$ & Thickness of piezoelectric layer $(\mathrm{mm})$ & 0.2 \\
$\omega_{n}$ & Natural frequency (rad/s) & 164.5 \\
$\theta$ & Electromechanical coupling efficient & $1.976 e-4$ \\
$C_{e}$ & Equivalent capacitance $(\mathrm{F})$ & $7.969 e-8$ \\
\hline
\end{tabular}

the electromechanical coupling coefficient, $V$ is the voltage on the load, $R$ is the load resistance, and $C_{e}$ is the piezoelectric equivalent capacitance.

Figure 2 displays the structure, circuits, and equivalent circuits for the system. The piezoelectric layers of one cantilever and the two cantilevers are connected to $R$ in parallel and serial way, respectively.

$C_{e}$, the piezoelectric equivalent capacitance, and $\theta$, the electromechanical coupling coefficient, can be expressed by the following equation [27]:

$$
\begin{gathered}
C_{e}=C_{p}=\frac{\varepsilon_{0} \varepsilon_{r} b L_{p}}{h_{p}} \\
\theta=e_{31}\left(h_{p}+h_{s}\right) b,
\end{gathered}
$$

where $C_{p}$ is the capacitance of a single piezoelectric layer, $e_{31}$ is the piezoelectric coefficient, $\varepsilon_{0}$ is the permittivity of vacuum, $\varepsilon_{r}$ is the relative dielectric constant, $L_{p}, b$, and $h_{p}$ are length, width, and height of the piezoelectric layer, and $h_{s}$ is height of the base layer.

\section{Experimental Study}

In this paper, the diameter of the small cylinder $(d)$ is $10 \mathrm{~mm}$, the diameter of the big cylinder $(D)$ is $40 \mathrm{~mm}$, the piezoelectric material is $\mathrm{PZT}-5 \mathrm{H}$, and the material of the sublayer is copper. Parameters of the piezoelectric device are shown as in Table 1. The experiment is carried out in a subsonic open circuit wind tunnel, where air is driven by a centrifugal fan powered by a $1.5 \mathrm{~kW}$ motor. The section of the testing piece is $300 * 300 \mathrm{~mm}^{2}$, and its length is $2000 \mathrm{~mm}$. Two piezoelectric cantilevers of the experimental facility clamp vertically the middle location of the testing piece for doing the VIV experiment. There is no change for the location of the piezoelectric device. A circular cylinder of $40 \mathrm{~mm}$ diameter is put upstream for making the WIV experiment, and space $S$ between two cylinders is adjusted by moving the bigger cylinder's position. 


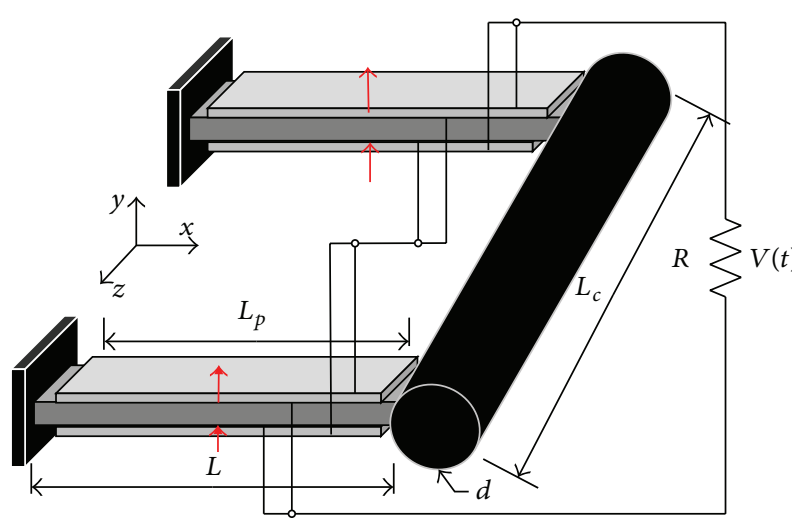

Piezoelectric layer Substructure

\begin{abstract}
个 Substructure
\end{abstract}
(a)

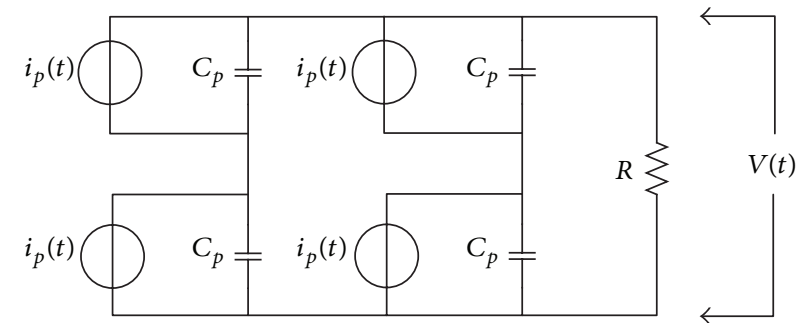

(b)

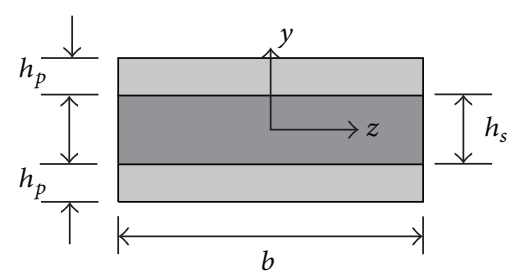

(c)

FIGURE 2: (a) Energy harvesting circuit connection, (b) equivalent circuit, and (c) cross-sectional view of a bimorph cantilever.

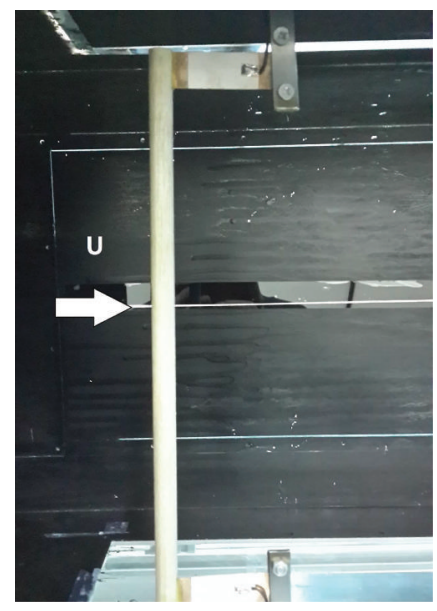

(a) VIV

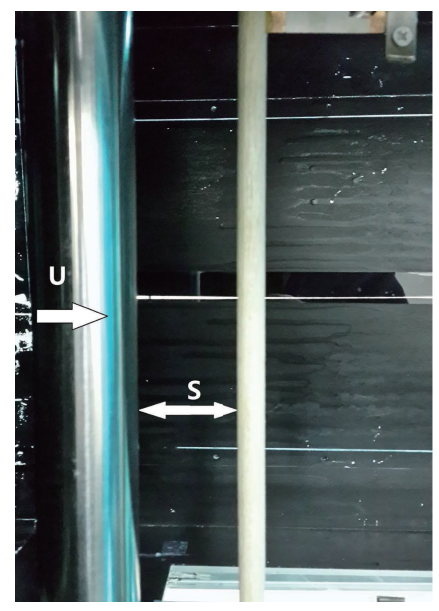

(b) WIV

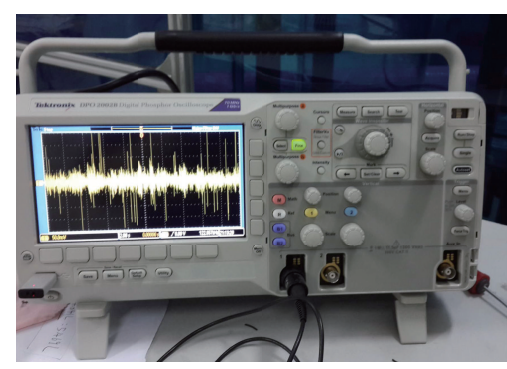

(c) Oscilloscope

FIgURE 3: Experiment setup of piezoelectric energy harvesting.

The voltage output from the load resistance is recorded with the oscilloscope of TEK2002B, and the main experimental device and its installation are shown as in Figure 3.

3.1. VIV Energy Harvesting Experiment. Four different load resistance values, $10^{4} \Omega, 10^{5} \Omega, 10^{6} \Omega$, and $10^{7} \Omega$, are selected for the VIV experiment. The experiment at a certain load resistance is done by gradually increasing the wind speed, and total four groups of experiments are done and corresponding voltage outputs are recorded, respectively. The range of wind speeds is $0.82 \leq U^{*} \leq 7.03$, where $U^{*}=U / f_{n} D$, with $U$ being the freestream velocity in $\mathrm{m} / \mathrm{s}$ and $f_{n}$ being the natural frequency in $\mathrm{Hz}$. Figure 4(a) shows that the voltage output reaches the maximum value when $U^{*}=5$; this indicates that the system is in lock-in status and the largest amplitude is obtained. Thus, the voltage increases with the load resistance. When the load resistance varies in the range of $10^{4} \sim 10^{5} \Omega$, the absolute voltage is lower and the amplification is small. 


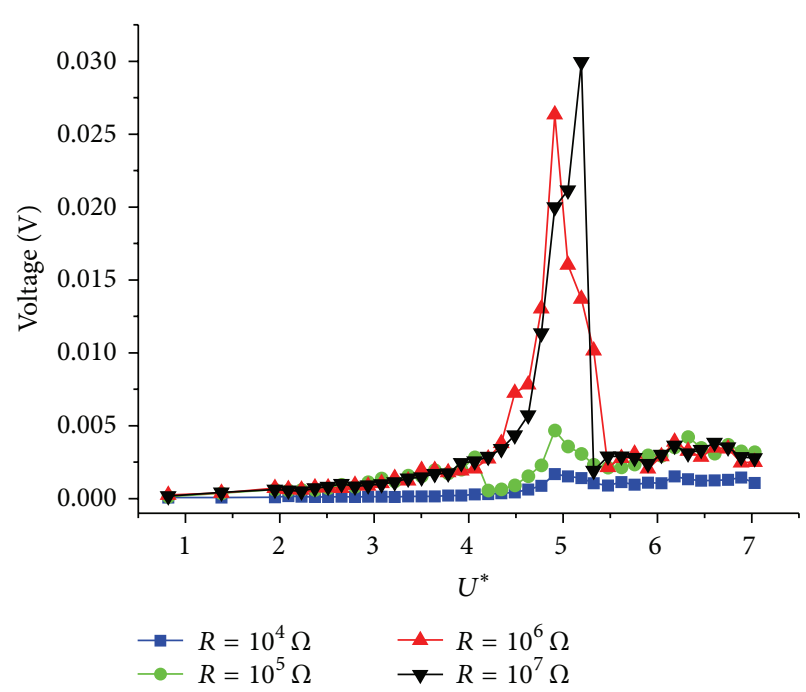

(a)

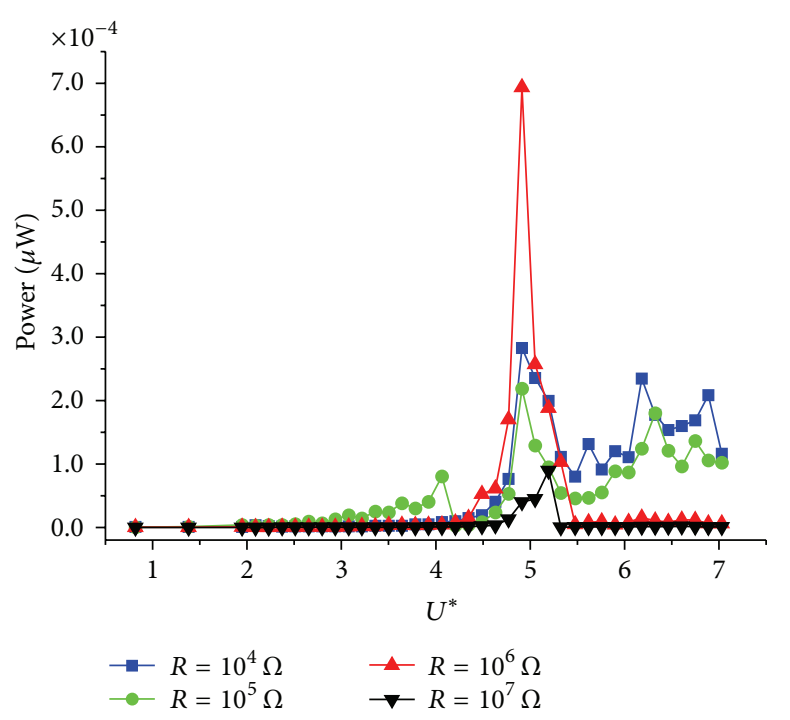

(b)

FIGURE 4: VIV response curves of the voltage and power at different load resistances.

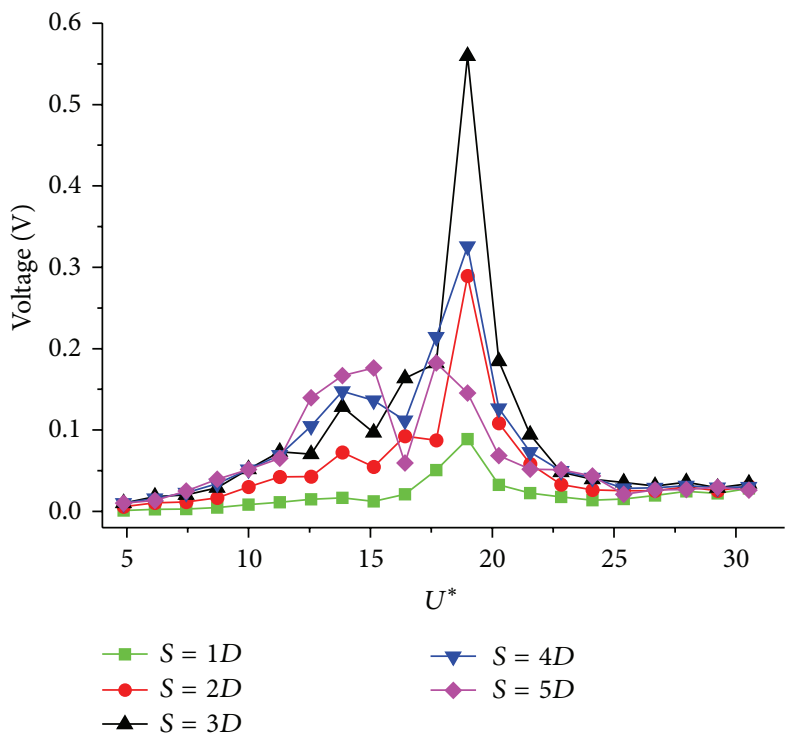

(a)

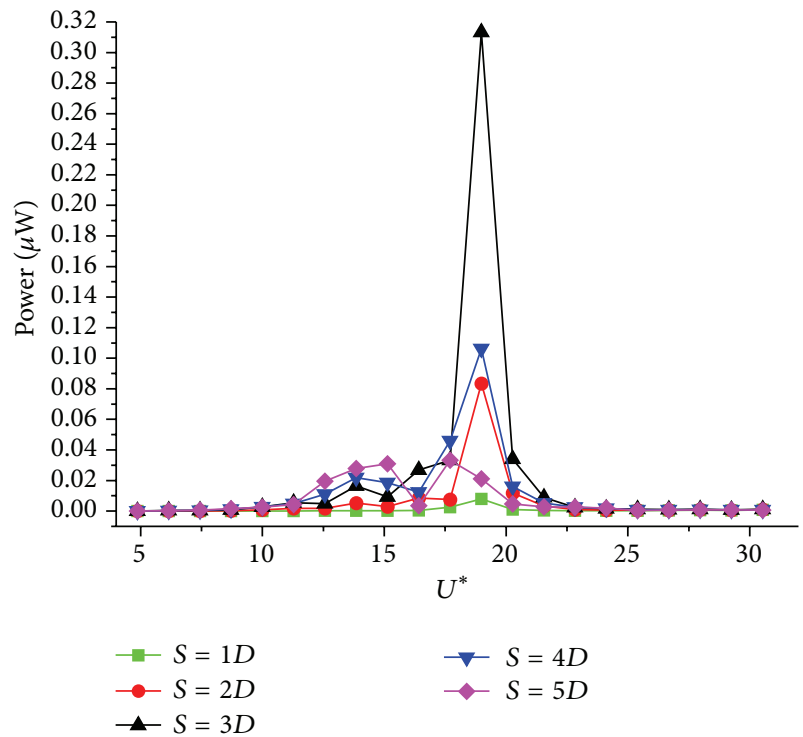

(b)

FIGURE 5: WIV response curves of the (a) voltage and (b) power at different load resistances.

But when the load resistance is up to $10^{6} \Omega$, there is a clear increase in the voltage output. Similarly, when $R$ is in the range of $10^{6} \sim 10^{7} \Omega$, the voltage output still increases, but the amplification narrows. Figure 4(b) shows that the maximum power of each load resistance happens near $U^{*}=5$, and when $R$ is $10^{6} \Omega$, the system's power reaches its maximum value.

3.2. WIV Energy Harvesting Experiment. The results of VIV experiments show that the power output at $R=10^{6} \Omega$ is the largest. So, the load resistance in the WIV experiments is fixed at $10^{6} \Omega$. By adjusting the location of the bigger cylinder, the value of $S$ changes between $1 D$ and $5 D$. For each $S$, experiments are done in the range of $2.3 \leq U^{*} \leq 31.8$, and the corresponding voltage outputs are recorded. Figure 5(a) shows that each $S$ reaches its maximum value when $U^{*} \approx$ 19 , which indicates that the wake shedding frequency of the bigger cylinder upstream is close to the natural frequency of the system at this time and the system is locked and the amplification is larger, so larger voltage output is obtained. With the increase of $S$, the voltage output of the system is up at first and then down. This is because the space flow does not develop fully at smaller $S$, so there is no strong vortex existing in the space. However, when $S$ continues to increase, the wake vortex also cannot play obvious coupling effect due 


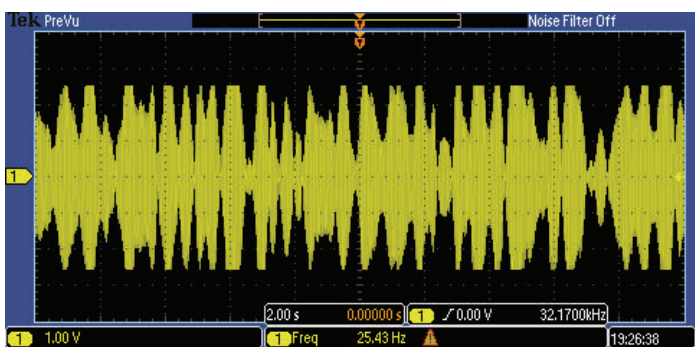

(a)

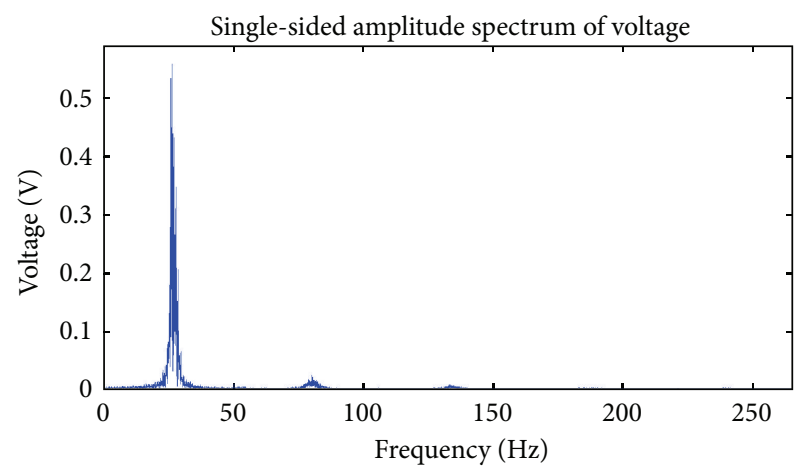

(b)

FIGURE 6: (a) WIV time traces of the voltage and (b) power spectra of voltage when $U^{*}=18.984$.

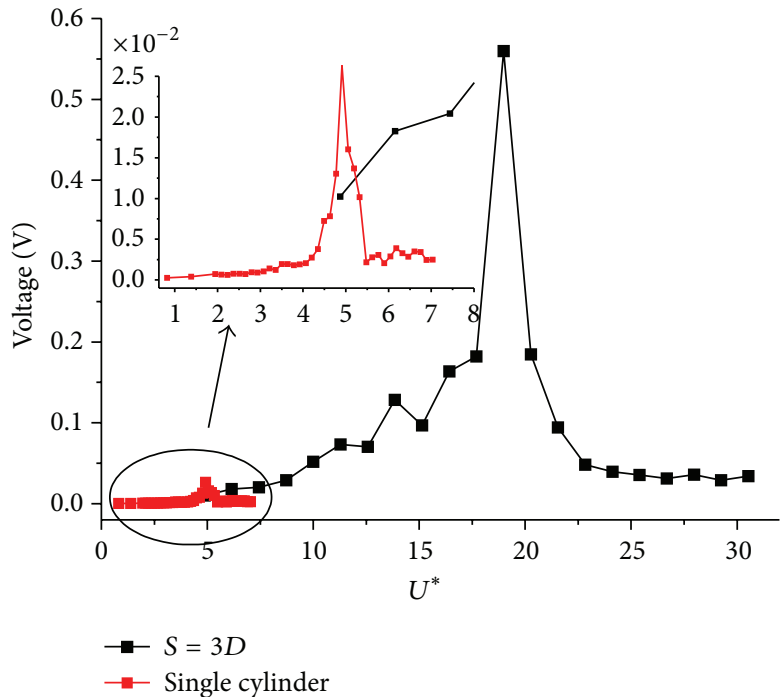

(a)

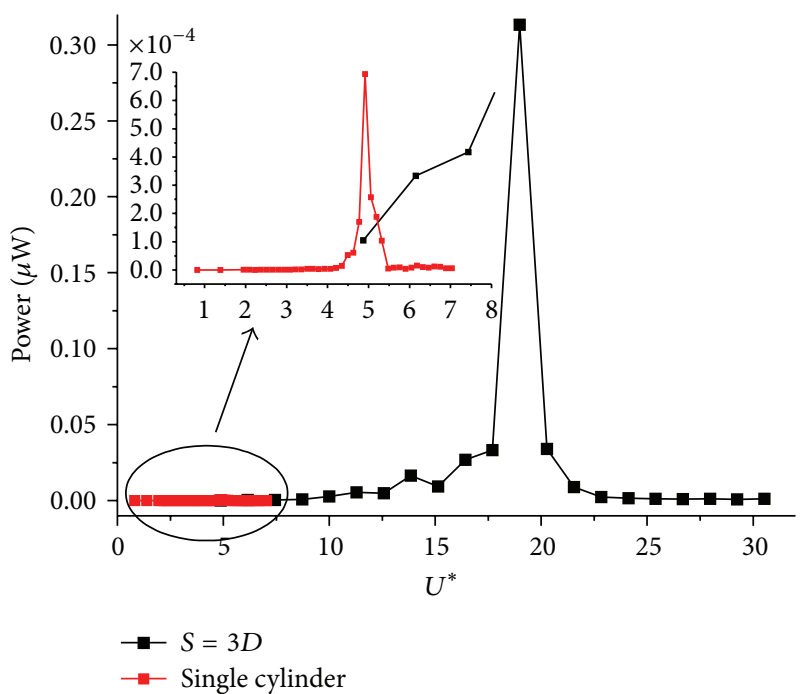

(b)

FIGURE 7: Comparison of (a) voltage output and (b) power output for WIV and VIV.

to the decrease of the dissipating intensity. The voltage output reaches the maximum value at $S=3 D$, which indicates that the wake produced by the bigger cylinder upstream has the strongest coupling effect with the smaller cylinder at this time, and the large amplification is attained. Because the load resistance is fixed, so the power output curve is similar to the voltage output and also reaches the maximum value at $S=3 D$, just as shown in Figure 5(b).

3.3. Discussions. Figure 6(a) shows the time history curve of voltage outputs recorded by oscilloscope in the WIV experimental at $U^{*}=18.984$. Figure $6(\mathrm{~b})$ is the spectrogram plot attained by carrying out FFT operation over the time history curve; the vibrational dominant frequency is $26.37 \mathrm{~Hz}$ which is very close to $f_{n}=26.18 \mathrm{~Hz}$. The vortex shedding frequency of the bigger cylinder upstream can be represented by $f=S_{t} U / D$. Because of $D=4 d$, if we assume that the Strouhal number $S_{t}$ is unchanged, then the vortex shedding frequency of VIV is four times as much as that of WIV under the same wind speed. Therefore, in order to make the vortex shedding frequency match with the natural frequency, the wind speed at WIV is needed to be higher.

Figure 7(a) shows that the lock-in region moves backward obviously, and the locked interval enlarges. The maximum value occurs at $U^{*}=18.984$ when $S=3 D$. For a single cylinder, the maximum value occurs at $U^{*}=4.913$. The ratio of the above two velocities is 3.86 , which indicates that the vibration of the smaller cylinder is controlled by the wake vortex shedding frequency of the bigger cylinder upstream at WIV. For the WIV case with fixed larger cylinder upstream and smaller cylinder downstream, there is a best gap which can make the effect of fluid-structure interaction be enhanced and obtain a larger amplification. For the piezoelectric energy harvesting, this feature of WIV is helpful for obtaining the higher voltage and power output.

\section{Conclusions}

This paper does the VIV experiment on the energy harvesting device with a circular cylinder and two piezoelectric beams, 
and the WIV experiment is done by putting a fixed bigger cylinder upstream to study the impacts on the piezoelectric energy harvesting by two types of flow-induced vibrations. The results of the VIV experiment show that the voltage output increases with the increase of the load resistance; there is a best load resistance for the power output. The results of the WIV experiment indicate that the vibration frequency of the smaller cylinder is controlled by the vortex shedding frequency of the bigger cylinder upstream, and there is a best gap between two cylinders for obtaining the maximum voltage and power output. Compared with VIV, the lock-in region of WIV wholly moves backward due to the bigger diameter of the cylinder upstream, which means slightly higher wind speed is needed to obtain the maximum power output. It is also found that the maximum power output of WIV is larger than that of VIV, so for the same energy harvesting device; WIV has much higher power generation capacity and can improve the piezoelectric output properties effectively.

\section{Competing Interests}

The authors declare that there is no conflict of interests regarding the publication of this paper.

\section{Acknowledgments}

This work was supported by Open Fund of Chongqing University, Key Laboratory of Low-Grade Energy Utilization Technologies and Systems (Grant no. LLEUTS-201610) and Open Fund of Jiangsu Engineering Research Center on Meteorological Energy Using and Control/C-MEIC, Nanjing University of Information Science \& Technology (Grant no. KCMEIC02).

\section{References}

[1] P. Muralt, "Ferroelectric thin films for micro-sensors and actuators: a review," Journal of Micromechanics and Microengineering, vol. 10, no. 2, pp. 136-146, 2000.

[2] W. Zhou, W. H. Liao, and W. J. Li, "Analysis and design of a selfpowered piezoelectric microaccelerometer," in Smart Structures and Materials 2005: Smart Electronics, MEMS, BioMEMS, and Nanotechnology, Proceedings of SPIE, pp. 233-240, San Diego, Calif, USA, May 2005.

[3] S. Roundy and P. K. Wright, "A piezoelectric vibration based generator for wireless electronics," Smart Materials and Structures, vol. 13, no. 5, pp. 1131-1142, 2004.

[4] D. J. Inman and B. L. Grisso, "Towards autonomous sensing," in Proceedings of the Smart Structures and Materials: Sensors and Smart Structures Technologies for Civil, Mechanical, and Aerospace Systems, vol. 6174, p. 61740T-T-7, San Diego, Calif, USA, February 2006.

[5] I. D. Capel, H. M. Dorrell, E. P. Spencer, and M. W. L. Davis, "The amelioration of the suffering associated with spinal cord injury with subperception transcranial electrical stimulation," Spinal Cord, vol. 41, no. 2, pp. 109-117, 2003.

[6] A. Erturk, W. G. R. Vieira, C. De Marqui Jr., and D. J. Inman, "On the energy harvesting potential of piezoaeroelastic systems," Applied Physics Letters, vol. 96, no. 18, Article ID 184103, 2010.
[7] C. De Marqui Jr., A. Erturk, and D. J. Inman, "Piezoaeroelastic modeling and analysis of a generator wing with continuous and segmented electrodes," Journal of Intelligent Material Systems and Structures, vol. 21, no. 10, pp. 983-993, 2010.

[8] J. C. De Marqui, W. G. R. Vieira, A. Erturk, and D. J. Inman, "Modeling and analysis of piezoelectric energy harvesting from aeroelastic vibrations using the doublet-lattice method," Journal of Vibration and Acoustics, vol. 133, no. 1, Article ID 011003, 9 pages, 2010.

[9] A. Abdelkefi, A. H. Nayfeh, and M. R. Hajj, "Modeling and analysis of piezoaeroelastic energy harvesters," Nonlinear Dynamics, vol. 67, no. 2, pp. 925-939, 2012.

[10] A. Abdelkefi, A. H. Nayfeh, and M. R. Hajj, "Design of piezoaeroelastic energy harvesters," Nonlinear Dynamics, vol. 68, no. 4, pp. 519-530, 2012.

[11] A. Abdelkefi, A. H. Nayfeh, and M. R. Hajj, "Enhancement of power harvesting from piezoaeroelastic systems," Nonlinear Dynamics, vol. 68, no. 4, pp. 531-541, 2012.

[12] A. Abdelkefi, M. R. Hajj, and A. H. Nayfeh, "Sensitivity analysis of piezoaeroelastic energy harvesters," Journal of Intelligent Material Systems and Structures, vol. 23, no. 13, pp. 1523-1531, 2012.

[13] S. Pobering and N. Schwesinger, "A novel hydropower harvesting device," in Proceedings of the International Conference on MEMS, NANO and Smart Systems (ICMENS '04), pp. 480-485, Banff, Canada, August 2004.

[14] O. Doaré and S. Michelin, "Piezoelectric coupling in energyharvesting fluttering flexible plates: linear stability analysis and conversion efficiency," Journal of Fluids and Structures, vol. 27, no. 8, pp. 1357-1375, 2011.

[15] S. Michelin and O. Doaré, "Energy harvesting efficiency of piezoelectric flags in axial flows," Journal of Fluid Mechanics, vol. 714, pp. 489-504, 2013.

[16] C. H. K. Williamson and R. Govardhan, "Vortex-induced vibrations," Annual Review of Fluid Mechanics, vol. 36, no. 1, pp. 413455, 2004.

[17] G. R. Ássi, J. R. Meneghini, J. A. Aranha et al., "Experimental investigation of flow-induced vibrations interference between two circular cylinders in tandem arrangements," in Proceedings of the ASME 24th International Conference on Offshore Mechanics and Arctic Engineering, American Society of Mechanical Engineers, pp. 273-277, 2005.

[18] G. R. S. Assi, P. W. Bearman, and J. R. Meneghini, "On the wake-induced vibration of tandem circular cylinders: the vortex interaction excitation mechanism," Journal of Fluid Mechanics, vol. 661, pp. 365-401, 2010.

[19] B. S. Carmo, S. J. Sherwin, P. W. Bearman, and R. H. J. Willden, "Flow-induced vibration of a circular cylinder subjected to wake interference at low Reynolds number," Journal of Fluids and Structures, vol. 27, no. 4, pp. 503-522, 2011.

[20] F. J. Huera-Huarte and M. Gharib, "Vortex- and wake-induced vibrations of a tandem arrangement of two flexible circular cylinders with far wake interference," Journal of Fluids and Structures, vol. 27, no. 5-6, pp. 824-828, 2011.

[21] K. M. Lam and A. P. To, "Interference effect of an upstream larger cylinder on the lock-in vibration of a flexibly mounted circular cylinder," Journal of Fluids and Structures, vol. 17, no. 8, pp. 1059-1078, 2003.

[22] H. Wang, W. Yang, K. D. Nguyen, and G. Yu, "Wake-induced vibrations of an elastically mounted cylinder located downstream of a stationary larger cylinder at low Reynolds numbers," Journal of Fluids and Structures, vol. 50, pp. 479-496, 2014. 
[23] H. D. Akaydin, N. Elvin, and Y. Andreopoulos, "The performance of a self-excited fluidic energy harvester," Smart Materials and Structures, vol. 21, no. 2, Article ID 025007, 2012.

[24] E. Molino-Minero-Re, M. Carbonell-Ventura, C. Fisac-Fuentes, A. Mànuel-Làzaro, and D. M. Toma, "Piezoelectric energy harvesting from induced vortex in water flow," in Proceedings of the IEEE International Instrumentation and Measurement Technology Conference (I2MTC '12), pp. 624-627, Graz, Austria, May 2012.

[25] A. Mehmood, A. Abdelkefi, M. R. Hajj, A. H. Nayfeh, I. Akhtar, and A. O. Nuhait, "Piezoelectric energy harvesting from vortexinduced vibrations of circular cylinder," Journal of Sound and Vibration, vol. 332, no. 19, pp. 4656-4667, 2013.

[26] M. Zhang, Y. Liu, and Z. Cao, "Modeling of piezoelectric energy harvesting from freely oscillating cylinders in water flow," Mathematical Problems in Engineering, vol. 2014, Article ID 985360, 13 pages, 2014.

[27] A. Erturk and D. J. Inman, "An experimentally validated bimorph cantilever model for piezoelectric energy harvesting from base excitations," Smart Materials and Structures, vol. 18, no. 2, Article ID 025009, 2009. 


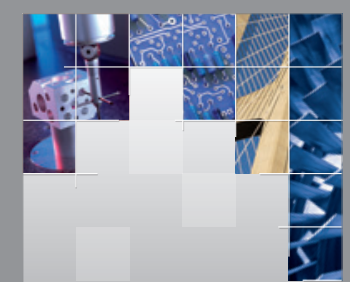

\section{Enfincering}
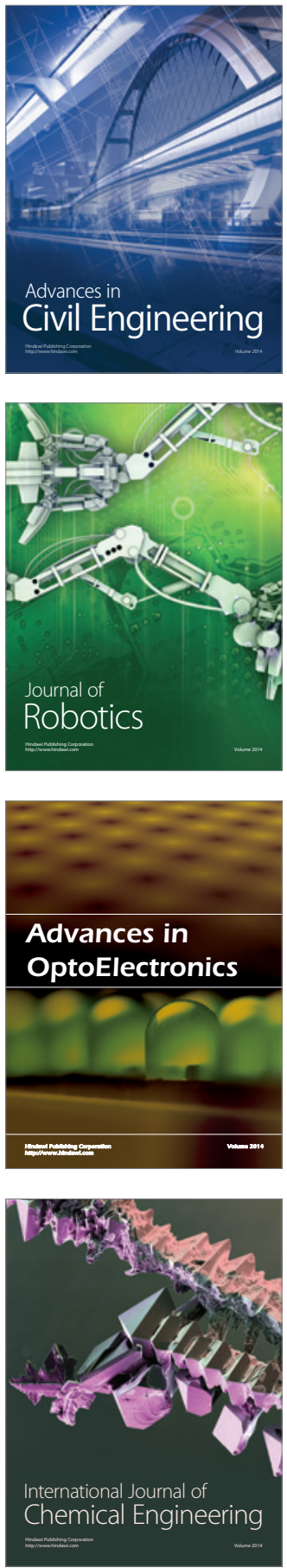

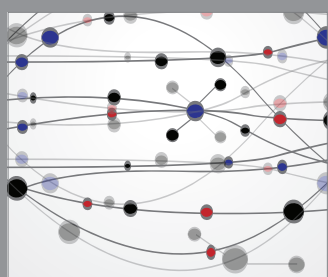

The Scientific World Journal

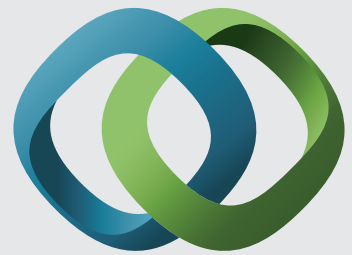

\section{Hindawi}

Submit your manuscripts at

http://www.hindawi.com
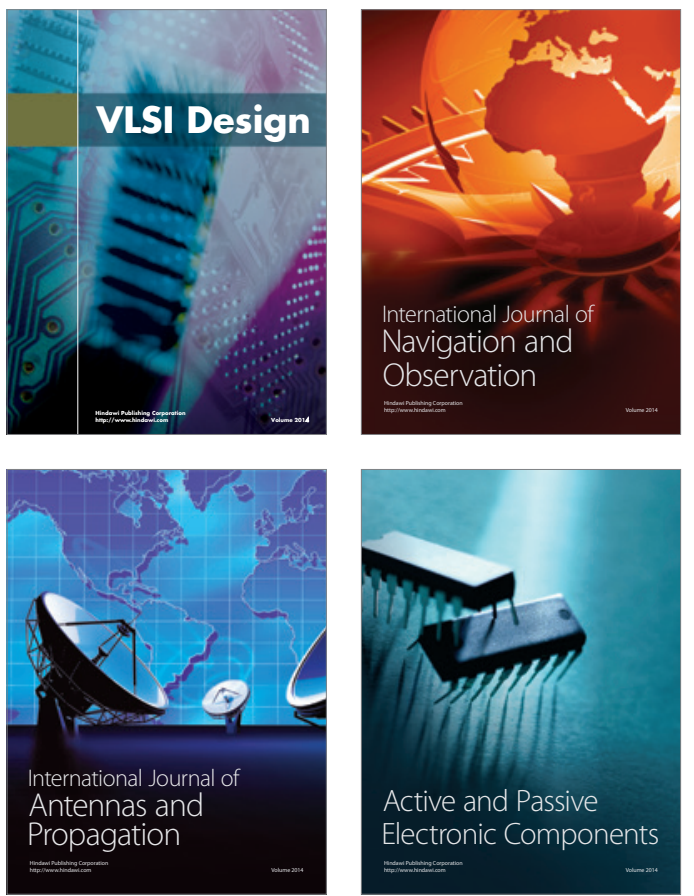
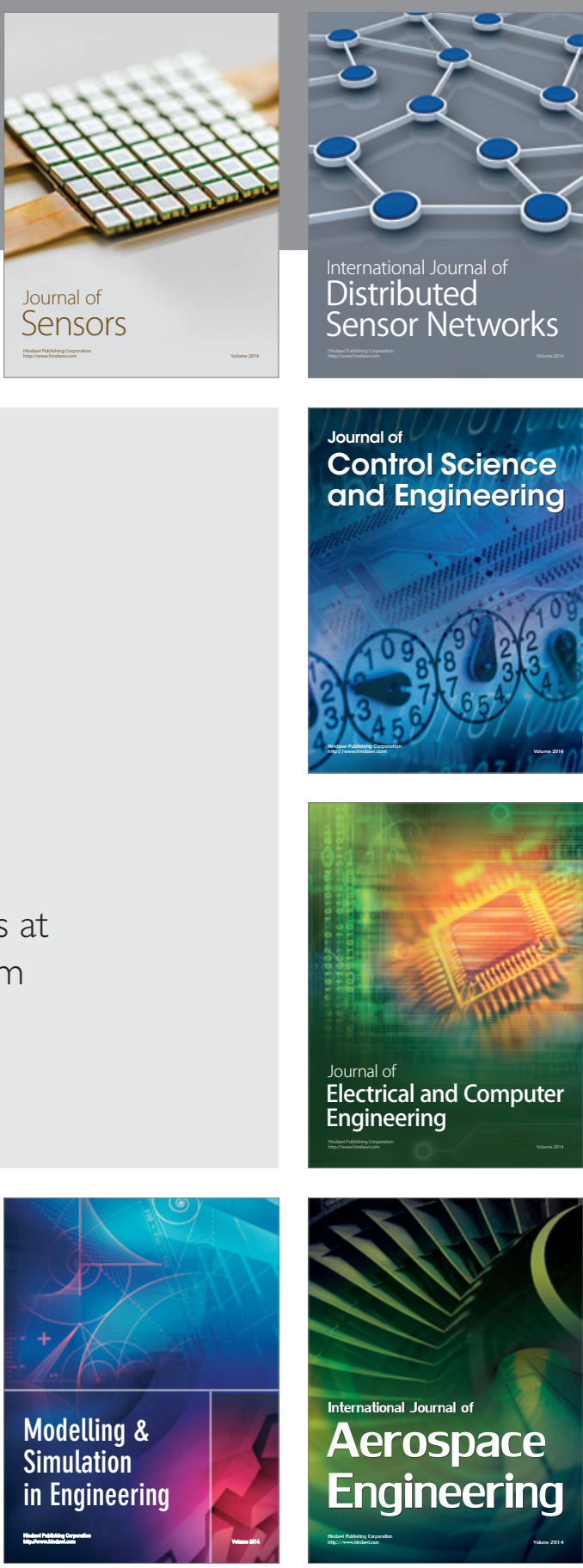

International Journal of

Distributed

Sensor Networks

Journal of

Control Science

and Engineering
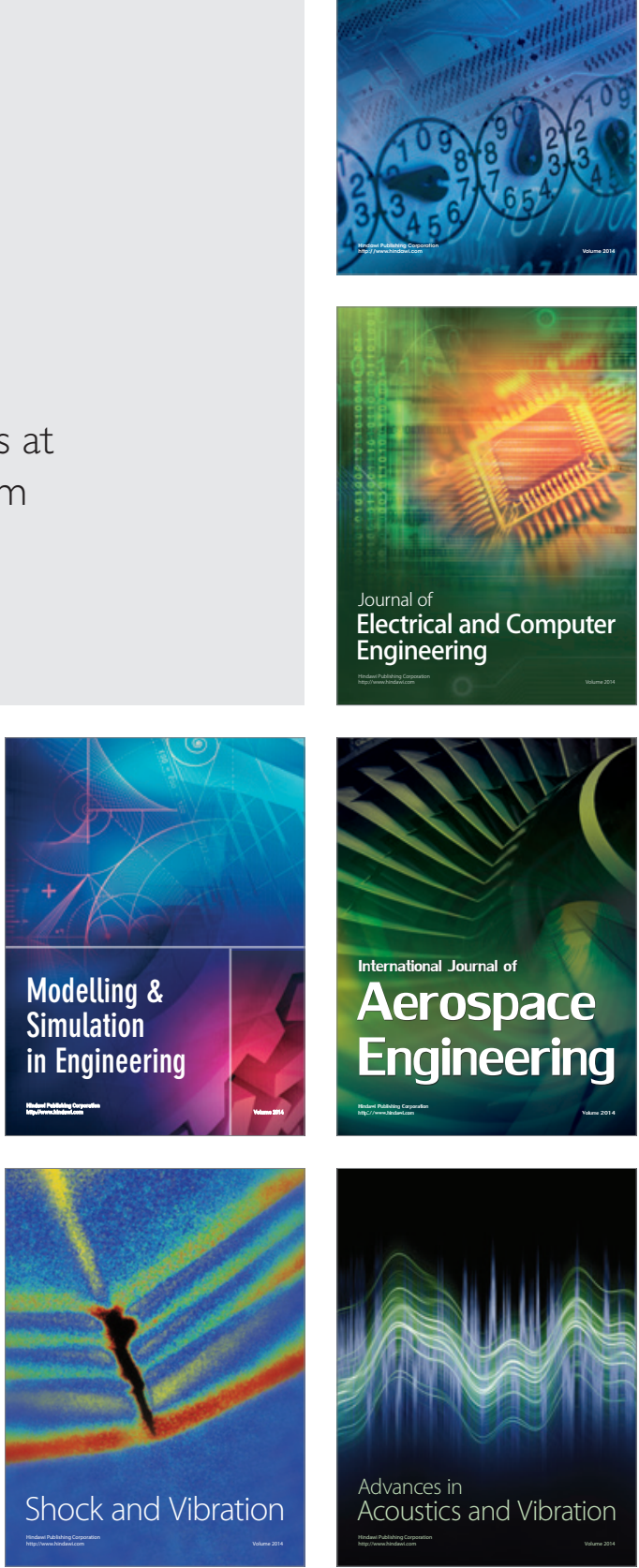